\title{
Multiple brushings with immediate Riu's stain via flexible fibreoptic bronchoscopy without fluoroscopic guidance in the diagnosis of peripheral pulmonary tumours
}

Cheng-Huei Lee, Chun-Hua Wang, Meng-Chih Lin, Thomas CY Tsao, Ray-Shee Lan, Ying-Huang Tsai, Han-Pin Kuo

\begin{abstract}
Background - Accurate diagnosis of peripheral pulmonary lesions usually relies on fluoroscopic guided procedures. As fluoroscopy is not routinely available in many respiratory units, an approach not using fluoroscopy but with a high diagnostic yield is highly desirable.

Methods - Immediate cytological examination of multiple brushings using Riu's stain, a modified Wright's stain, was performed in 38 patients with peripheral pulmonary lesions not visible at bronchoscopy. The results were compared with the final diagnoses determined by histological examination or subsequent Papanicolaou staining of cytological specimens and clinical course.

Results - Of the 38 patients 29 were subsequently confirmed to have a malignant tumour. Our method provided a diagnosis of malignancy in $86 \%$ of these lesions. The accuracy $(91 \%)$ and sensitivity $(88 \%)$ were higher for lesions $>3 \mathbf{c m}$ in diameter than for those of diameter $\leqslant 3 \mathrm{~cm}(87 \%$ and $83 \%)$. There were no false positive results. The 29 lesions correctly diagnosed as malignant by Riu's stain required significantly fewer brushings (mean (SD) 3 (2)) than the nine benign lesions (5 (4)). Conclusions - This technique provides a high diagnostic yield, avoids the need for fluoroscopy, and is probably safer than percutaneous biopsy.

(Thorax 1995;50:18-21)
\end{abstract}

Keywords: lung tumour, fibreoptic bronchoscopy, Riu's stain, bronchial brushing.

Department of Thoracic Medicine, Chang Gung Memorial Hospital, 199 Tun-Hwa North Road, Taipei,

Taiwan

C-H Lee

C-H Wang

$M-C$ Lin

T C Y Tsao

R-S Lan

Y-H Tsai

H-P Kuo

Reprint requests to:

Dr C-H Lee.

Received 28 March 1994

Returned to authors

2 June 1994

Revised version received

1 August 1994

Accepted for publication

17 October 1994 timal results, but the diagnostic rate of brushing with fluoroscopy has remained low (30-78\%). ${ }^{1245}$ Repeated brush biopsy has been reported to achieve a higher diagnostic yield. ${ }^{6}$ Transthoracic fine needle aspiration (FNA) biopsy under fluoroscopy or computed tomographic (CT) scanning could provide a diagnostic rate of over $90 \%$ for malignant nodules. ${ }^{78}$ However, complications including pneumothorax and intrapulmonary bleeding occur in $25-36 \%$ and $5-10 \%$ of cases, respectively. ${ }^{7-10}$ In addition, fluoroscopy or CT scanning is not routinely available in many respiratory units. An approach which can give a high diagnostic yield without fluoroscopy, together with a low incidence of serious complications, is desirable. We propose a new method using repeated bronchial brushings via the fibreoptic bronchoscope with immediate cytological examination using Riu's stain, " a method modified from Wright's stain. The stain has the advantage of a long shelf life and short staining time (two minutes including fixation).

\section{Methods}

SUBJECTS

Thirty eight patients (23 men) aged 31-83 years who presented with pulmonary masses on their chest radiographs to the pulmonary service of Chang Gung Memorial Hospital, Taipei, Taiwan, between July 1990 and September 1993 were studied prospectively. The size of the lesion on the radiograph was determined as the largest diameter and was agreed upon by two chest physicians. Of the 38 patients, 15 had masses of $\leqslant 3 \mathrm{~cm}$ in diameter while the other 23 had lesions $>3 \mathrm{~cm}$. In each patient the peripheral pulmonary mass was invisible at bronchoscopy.

Twenty nine of the 38 patients $(76 \%)$ were subsequently proved to have a malignancy by histological or Papanicolaou's cytological examination and clinical course. Of these 29 patients, one had carcinoma of the colon with a lung metastasis, and the other 28 all had a bronchogenic carcinoma which was confirmed by thoracotomy in 15 patients, by cytological examination and clinical course in 10 , and by percutaneous transthoracic biopsy in four. Nine patients $(24 \%)$ had benign lesions confirmed by histological examination in seven, including two with an organising pneumonia, three with caseating granuloma, one with neurofibroma, and one with a sclerosing haemangioma. The remaining two cases showed no change in size of the lesion over a two year follow up. The anatomical distribution of the peripheral pulmonary lesions was right upper lobe (10), right middle lobe (two), right lower lobe (10), left 
upper lobe (six), lingula (two), and left lower lobe (eight). The most common location for malignant lesions was the right upper lobe.

\section{STUDY DESIGN}

The probable location of the tumour was determined initially by conventional posteroanterior and lateral chest radiography or computed tomographic scanning. Flexible fibreoptic bronchoscopy (Olympus BF-P10) without fluoroscopic guidance was then performed transnasally using $2 \%$ xylocaine for local anaesthesia. Each patient had supplemental oxygen administered routinely by nasal cannula and was monitored for heart rate and rhythm. Midazolam $(0.05-0.07 \mathrm{mg} / \mathrm{kg}$ intravenously) was occasionally used to reduce anxiety in patients receiving a prolonged bronchoscopy. At the appropriate predetermined segment several orifices of the subsegmental bronchi were visualised and the tip of the bronchoscope advanced as far as possible. Bronchial brushings were taken with a nylon brush (Olympus BC-1J) from the subsegmental bronchus considered likely to contain the lesion, advancing it as far as possible. The whole bronchoscope with the brush was then withdrawn and the specimen was spread immediately onto two glass slides which were separated thereafter. One slide was air dried and immediately stained with Riu's stain. The other was immersed in 95\% alcohol and submitted for the standard Papanicolaou's stain. The specimen with Riu's stain was immediately evaluated by light microscopy. If the specimen was negative for malignancy the procedure was repeated on another subsegmental bronchus until all the suspected subsegmental bronchi had been assessed. When all bronchial brushings were negative with Riu's stain the pulmonary lesion was considered to be benign. If the Riu's stain specimen was positive, the lesion was considered to be malignant. The judgement was later confirmed by comparing the findings with those of the histological examination of any subsequent surgical biopsy specimens, the Papanicolaou stain brush cytological examination, or the clinical course. Specimens were interpreted by the cytopathologist without prior knowledge of the histological result. Histological specimens were stained with haematoxylin and eosin and also interpreted by independent pathologists without prior knowledge of the clinical course.

\section{PREPARATION AND STAINING METHOD OF RIU'S} STAIN

Riu's stain was modified from Wright's stain by Professor Chin-Hui Riu in 1965.1 The staining solutions consist of two solutions, A and B. ${ }^{12}$

\section{Solution $A$}

This comprises $0 \cdot 17-0 \cdot 18 \mathrm{~g}$ of eosin $\mathrm{Y}, 0.07 \mathrm{~g}$ of medicinal extra pure methylene blue, and $100 \mathrm{ml}$ of absolute methanol. Eosin Y is dissolved in the methanol and the methylene blue is added. The solution is shaken, allowed to stand for 24 hours, and filtered before use. Some precipitation may sometimes reappear 2-3 days after the first filtering, in which case the solution is filtered again and kept in a clear glass bottle. It may be preserved for a long time if the bottle is tightly stoppered.

\section{Solution B}

This consists of $0.7 \mathrm{~g}$ medicinal extra pure methylene blue, $0.6-0.7 \mathrm{~g}$ azure I, $6.25 \mathrm{~g}$ $\mathrm{KH}_{2} \mathrm{PO}_{4}, \quad 12.6 \mathrm{~g} \quad \mathrm{Na}_{2} \mathrm{HPO}_{4} \cdot 12 \mathrm{H}_{2} \mathrm{O}$ ), and $500 \mathrm{ml}$ distilled water. The $\mathrm{Na}_{2} \mathrm{HPO}_{4} \cdot 12 \mathrm{H}_{2} \mathrm{O}$ and $\mathrm{KH}_{2} \mathrm{PO}_{4}$ are first dissolved in distilled water, while azure $\mathrm{I}$ is ground in a mortar and added gradually to the buffer solution. Azure $\mathrm{I}$ is dissolved maximally by this means. The solution is put into a flask and the methylene blue added, shaken vigorously, and filtered after 24 hours. It can be preserved for a long time. The staining effect increases with aging.

\section{Staining method}

Staining is carried out after the smeared specimen has dried in air. Two to three drops of solution $\mathrm{A}$ are put on the slide for 30 seconds, then two to three drops of solution $B$ are added for 60 seconds and the slide is rinsed in running water. The total time for staining is only 90 seconds.

\section{STATISTICAL ANALYSIS}

The unpaired $t$ test was used to compare the numbers of bronchial brushings required for benign or malignant lesions, and tumour size. Differences of location were compared by ANOVA, and the correlation between the number of bronchial brushings and the sensitivity for malignancy by linear regression. A $p$ value of $<0.05$ was considered statistically significant.

\section{Results}

Using repeated bronchial brushings and immediate Riu's stain 25 patients were classified as having a malignant tumour and 13 were considered to be benign. When compared with the final diagnosis the overall diagnostic accuracy rate was $89 \%$. The diagnostic sensitivity for malignancy in patients with a tumour $>3 \mathrm{~cm}$ in diameter was $88 \%$, which was better than that in patients with a tumour of $\leqslant 3 \mathrm{~cm}(83 \%)$ (table 1). The overall diagnostic yield for tumours of $\leqslant 3 \mathrm{~cm}$ compared with those of $>3 \mathrm{~cm}$ in diameter was $87 \%$ and $91 \%$, respectively. No false positive diagnosis of malignancy was made in patients with benign lesions. The false negative rate for malignancy was $14 \%$. In the four lesions which were proved to be false negative two were $\leqslant 3 \mathrm{~cm}$ and two were $>3 \mathrm{~cm}$ in diameter.

Of the 34 subjects who were accurately identified, a positive diagnosis of malignant disease was established with a mean (SD) of 3 (2) bronchial brushings, which was significantly less than the 5 (4) brushings required for those 
Table 1 Effect of tumour size on the diagnosis

\begin{tabular}{|c|c|c|c|c|c|c|}
\hline \multirow{2}{*}{$\begin{array}{l}\text { Riu's stain } \\
\text { method }\end{array}$} & \multicolumn{2}{|c|}{ Tumour size $\leqslant 3 \mathrm{~cm}(n=15)$} & \multicolumn{2}{|c|}{ Tumour size $>3 \mathrm{~cm}(n=23)$} & \multicolumn{2}{|l|}{ Total $(n=38)$} \\
\hline & Malignant & Benign & Malignant & Benign & Malignant & Benign \\
\hline $\begin{array}{l}\text { Malignant } \\
\text { Benign } \\
\text { Total }\end{array}$ & $\begin{array}{r}10 \\
2 \\
12\end{array}$ & $\begin{array}{l}0 \\
3 \\
3\end{array}$ & $\begin{array}{r}15 \\
2 \\
17\end{array}$ & $\begin{array}{l}0 \\
6 \\
6\end{array}$ & $\begin{array}{r}25 \\
4 \\
29\end{array}$ & $\begin{array}{l}0 \\
9 \\
9\end{array}$ \\
\hline $\begin{array}{l}\text { Sensitivity for } \\
\text { malignancy }\end{array}$ & $10 / 12(83 \%)$ & & $15 / 17(88 \%)$ & & $25 / 29(86 \%)$ & \\
\hline
\end{tabular}

Overall diagnostic accuracy rate $87 \%$ for tumour size $\leqslant 3 \mathrm{~cm}, 91 \%$ for tumour size $>3 \mathrm{~cm}$, and $89 \%$ for total.

Table 2 Mean (SD) numbers of brushings needed to achieve a diagnosis comparing benign and malignant tumours, tumour size, and different locations

\begin{tabular}{|c|c|c|c|c|c|c|}
\hline & \multirow{2}{*}{$\begin{array}{l}\text { Malignant } \\
(n=25)\end{array}$} & & \multirow{2}{*}{$\begin{array}{l}\text { Benign } \\
(n=9)\end{array}$} & & \multicolumn{2}{|c|}{ Tumour size } \\
\hline & & & & & $\begin{array}{l}\leqslant 3 \mathrm{~cm} \\
(n=14)\end{array}$ & $\begin{array}{l}>3 \mathrm{~cm} \\
(n=20)\end{array}$ \\
\hline \multirow[t]{3}{*}{ No. of brushings } & \multicolumn{2}{|l|}{$3(2)$} & \multicolumn{2}{|l|}{$5(4)^{*}$} & $4(2)$ & $4(3)$ \\
\hline & \multicolumn{6}{|l|}{ Location } \\
\hline & $\begin{array}{l}R U L \\
(n=8)\end{array}$ & $\begin{array}{l}R M L \\
(n=1)\end{array}$ & $\begin{array}{l}R L L \\
(n=10)\end{array}$ & $\begin{array}{l}L U L \\
(n=5)\end{array}$ & $\begin{array}{l}\text { Lingula } \\
(n=2)\end{array}$ & $\begin{array}{l}L L L \\
(n=8)\end{array}$ \\
\hline No. of brushings & $2(1)$ & 14 & $3(1)$ & $4(3)$ & 3,5 & $4(2)$ \\
\hline
\end{tabular}

RUL, $L U L=$ right and left upper lobes, RLL, $L L L=$ right and left lower lobes, $R M L=$ right middle lobe.

${ }^{*} \mathrm{p}<0.05$ compared with malignant group.

with benign lesions $(p<0 \cdot 05)$. There was no significant difference in the numbers of bronchial brushings required based on tumour size or anatomical location (table 2 ).

In diagnosing malignancy the sensitivity of the first brushing was only $21 \%$, but increased progressively to $86 \%$ with an increasing number of samples $(r=0.89, \mathrm{p}<0.02)$. The same tendency was observed for both small $(\leqslant 3 \mathrm{~cm}$, $r=0.88, \mathrm{p}<0.05)$ and large masses $(>3 \mathrm{~cm}, r=$ $0.99, \mathrm{p}<0.001$ ) (table 3).

There were no complications such as pneumothorax or massive haemoptysis. The time for each cytological examination after each brushing was less than five minutes. Patients could rest between brushings and most of the procedures were completed within one hour. Even for the patient who received 14 brushings the procedure took just one and a half hours. There were no serious arrhythmias and the procedure was well tolerated by our patients who all received supplemental oxygen during the bronchoscopy.

There were no false positive cytological results from the Riu's stain and $80 \%$ of the cell type diagnoses from the Riu's stain interpretations were consistent with the final histological diagnoses. The cytological diagnosis matched the histological diagnosis in all seven cases of squamous cell carcinoma, five of six cases

Table 3 Cumulative sensitivity of bronchial brushing numbers for diagnosing a malignancy based on tumour size

\begin{tabular}{lccc}
\hline Bruhing no. & \multicolumn{2}{c}{ Tumour size } & \\
\cline { 2 - 3 } & $\begin{array}{c}\leqslant \mathrm{cm}^{*} \\
(n=12)\end{array}$ & $\begin{array}{l}>3 \mathrm{~cm}^{* *} \\
(n=17)\end{array}$ & $\begin{array}{l}\text { Total* } \\
(n=29)\end{array}$ \\
\hline 1 & $3(25 \%)$ & $3(18 \%)$ & $6(21 \%)$ \\
2 & $6(50 \%)$ & $6(35 \%)$ & $12(41 \%)$ \\
3 & $8(67 \%)$ & $10(59 \%)$ & $18(62 \%)$ \\
4 & $8(67 \%)$ & $13(76 \%)$ & $21(72 \%)$ \\
5 & $9(75 \%)$ & $15(88 \%)$ & $24(83 \%)$ \\
$>5$ & $10(83 \%)$ & $15(88 \%)$ & $25(86 \%)$ \\
\hline
\end{tabular}

${ }^{*} \mathrm{p}<0.05,{ }^{* *} \mathrm{p}<0.001$. of adenocarcinoma, and none of two cases of bronchoalveolar cell carcinoma.

\section{Discussion}

Our results show that, even without fluoroscopic guidance, repeated bronchial brushings with immediate cytological examination by Riu's stain can yield a diagnosis of malignancy in $86 \%$ of peripheral tumours. This is equal to, or higher than, that of previous studies using fluoroscopy to assist in the harvest of bronchial brushngs $(30-86 \%)^{12561314}$ and superior to some without fluoroscopic guidance (around $28 \%) .^{1516}$

Our method requires minimal equipment and decreases the risk of radiological exposure, not only to the patient but also to the bronchoscopist and technician. Furthermore, the diagnostic yield from peripheral lung lesions $<3 \mathrm{~cm}$ in diameter was improved with our technique. Two of the false negative results from tumours located too peripherally to be reached by our technique were finally identified as malignant by a percutaneous transthoracic echo-guided biopsy. For those tumours situated truly peripherally transthoracic fine needle aspiration (FNA) may therefore be considered an alternative approach. Fibreoptic bronchoscopy using our technique and transthoracic FNA biopsy are, of course, complementary in the diagnosis of lesions not visible at bronchoscopy.

Riu's stain has been used routinely by many hospitals in Taiwan for blood smears and other cytological examinations. ${ }^{1217}$ It is simple and rapid and enables the bronchoscopist to sample each suspected subsegmental bronchi sequentially, with only a minimal delay between repeated brushings should the previous stained sample prove negative for malignant cells. Immediate cytological interpretation has the advantage of determining the adequacy of the specimens and thereby improving diagnostic accuracy and yield..$^{18}$ 
Diff-Quik stain is a popular rapid stain for FNA cytology. ${ }^{1820}$ Like Riu's stain the smear is air dried and the three step procedure takes about 20-30 seconds. Other stains such as toluidine blue are commonly used for FNA cytology elsewhere. ${ }^{91}$ These methods also offer the advantage of a rapid diagnosis and can be applied to our repeat brushing method.

In conclusion, without fluoroscopic guidance our method of repeated bronchial brushings with immediate Riu's stain and cytological examination showed a higher diagnostic accuracy for peripheral lung lesions than the method using fluoroscopy. It is probably safer than percutaneous biopsy because of lower radiological exposure, and fewer complications such as pneumothorax and bleeding have been reported. This technique provides an easy, time saving alternative approach when fluoroscopy is not available or is contraindicated.

The authors are grateful to Professor Yun-Fan Liaw for his suggestions and careful review of the manuscript.

1 Zavala DC. Diagnostic fiberoptic bronchoscopy: techniques and results of biopsy in 600 patients. Chest 1975;68:12-9.

2 Solomon DA, Solliday NH, Gracey DR. Cytology in fiberoptic bronchoscopy: comparison of bronchial brushing, washing and post-bronchoscopy sputum. Chest 1974;65: 616-9.

3 Arroliga AC, Matthay RA. The role of bronchoscopy in lung cancer. Clin Chest Med 1993;14:87-8.

4 Jay SJ, Wehr K, Nicholson DP, Smith AL. Diagnostic sensitivity and specificity of pulmonary cytology: comparison of techniques used in conjunction with flexible fiberoptic bronchoscopy. Acta Cytol 1980;24:304-12.

5 Lam WK, So SY, Hsu C, Yu DY. Fiberoptic bronchoscopy in the diagnosis of bronchial cancer: comparison of washings, brushings and biopsies in central and peripheral tumours. Clin Oncol 1983;9:35-42.

6 Popp W, Merkle M, Schreiber B, Rauscher H, Ritschka L, Zwick $\mathrm{H}$. How much brushing is enough for the diagnosis of lung tumors? Cancer 1992;70:2278-80.

7 Khouri NF, Stitik FP, Erozan YS, Gupta PK, Kim WS, et $a l$. Transthoracic needle aspiration biopsy of benign and

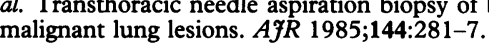

8 Salazar AM, Westcott JL. The role of transthoracic needle biopsy for the diagnosis and staging of lung cancer. Clin Chest Med 1993;14:99-110.

9 Perimutt LM, Johnston WW, Dunnick NR. Percutaneous ransthoracic needle aspiration: a review. $A f R$ 1989;152: 451-5.

10 Wallace JM, Deutsch AL. Flexible fiberoptic bronchoscopy and percutaneous needle lung aspiration for evaluating the solitary pulmonary nodule. Chest $1982 ; 81: 665-71$.

$11 \mathrm{Riu} \mathrm{CH}$. On the studies of the methods of staining blood film (Romanowsky's system). $\mathcal{F}$ Nigada Med Assoc June film (Romanowsky's system). F Nigada Med

12 Lee CH, Lan RS, Tsai YH, Chiang YC, Wang WJ. Riu's stain in the diagnosis of pulmonary cryptococcosis: in troduction of a new diagnostic method. Chest 1988;93: 467-70.

13 Stringfield JT, Markowitz DJ, Bentz RR, Welch MH, Weg JG. The effect of tumor size and location on diagnosis by fiberoptic bronchoscopy. Chest 1977;72:474-6.

14 Zavala DC, Richardson RH, Mukeriee PK, Rossi NP, Bedell GN. Use of the bronchofiberscope for bronchial brush biopsy: diagnostic results and comparison with other biopsy: diagnostic results and comparison
brushing techniques. Chest 1973;63:889-92.

15 Kvale PA, Bode FR, Kini S. Diagnostic accuracy in lung cancer: comparison of techniques used in association with flexible fiberoptic bronchoscopy. Chest 1976;69:752-7.

16 Mak VHF, Johnston IDA, Hetzel MR, Grubb C. Value of washings and brushings at fiberoptic bronchoscopy in the diagnosis of lung cancer. Thorax 1990;45:373-6.

17 Yu SC, Lin PW, Liaw KY, Wang SM, Wei TC, Chen KM et al. Fine needle aspiration cytology of breast tumors. $\mathcal{F}$ et al. Fine needle aspiration cytology
Formosan Med Assoc 1985;84:707-34.

18 Silverman JF, Frable WJ. The use of the Diff-Quik stain in the immediate interpretation of fine needle aspiration biopsies. Diagn Cytopathol 1990;6:366-9.

19 Park HY, Yokota S, Teplitz RL, Shaw SL, Werner JL. Rapid staining techniques employed in fine needle aspirations of the lung. Acta Cytol 1981;25:178-84.

20 Duguid HL, Wood RAB, Irving AD, Preece PE, Cuschieri A. Needle aspiration of the breast with immediate reporting of material. BMF 1979;2:185-7.

21 Harter LP, Moss AA, Goldberg HI, Gross BH. CT-guided fine-needle aspirations for diagnosis of benign and malignant disease. $A \Im R$ 1983;140:363-7. 